\title{
Energy Dispersive Spectroscopy (EDS) Measurements of Sulfur Uptake in Pigeon Bone Associated with the Intake of Slag, and the Induction of Homocystinuria
}

\author{
E. Galiano*, M. Lapointe \\ Department of Physics, Laurentian University, 935 Ramsey Lake Rd., Sudbury Ontario, P3E 2C6, Canada.
}

\section{ART I C LEDETAILS}

Article history:

Received 06 June 2020

Accepted 18 June 2020

Available online 28 June 2020

\section{Keywords:}

EDS

Sulfur Uptake

Bone Metabolism

Homocystinuria

\begin{abstract}
A B S T RA C T
Slag from nickel smelting operations over the last 150 years has been deposited over vast areas of soil around the Sudbury basin in Ontario, Canada. This metal-rich material containing a $2.50 \pm 0.50 \%$ sulfur concentration by mass, when ingested, has the potential to effect important physiological changes in biological systems. In this work, we measure the uptake of sulfur in the tibio-tarsal bones of pigeons (Columbia Livia Domestica) subjected to a slag-based diet, using energy dispersive spectroscopy (EDS). A sulfur-rich diet is associated with homocystinuria, a condition which impairs the conversion of methionine to cysteine. Among the bony changes associated with the condition are osteopenia, loss of calcium, compromised mechanical integrity, and increased resorption. In previous work, we have detected and quantified all of these changes, and thus propose that the results of the present work support the conclusion that a sulfur-rich, slag-based diet, can induce homocystinuric condition in this avian species.
\end{abstract}

\section{Introduction}

Slag tailings containing high concentrations of base metals from over 150 years of continuous nickel smelting operations in the Sudbury, Ontario basin in Canada, have become ubiquitous in the soil. Among its engineering uses are road construction, laying of railway beds, bridge building, construction of retaining buttresses, break walls, causeways, and dikes, surface stabilization of inclines and ravines, production of cement and concrete aggregates, and occasional use as a base substrate for parking lots and airport runways $[1,2]$. This material rich in heavy metals - particularly iron - upon dietary ingestion has the potential to effect profound physiological changes in the bony structures of biological systems. Micro chemical analysis has revealed that Sudbury basin nickel recovery slag contains approximately $2.50 \pm 0.50 \%$ sulfur concentration by weight [3]. Granivorous species depend on a continuous uptake of pebble-sized grit to assist in the grinding functions of the gizzard, and routinely obtain such pebbles from available sources within their home range environment. In this work, we measured the sulfur uptake in the tibio-tarsal bones of pigeons (Columbia Livia Domestica) subjected to Sudbury basin dietary slag ingestion. The specimens were fed slag-based grit for a period of 1 year and their tibio-tarsal bones were harvested for analysis. A control group was fed a slag-free diet. Selection, feeding, husbandry, and sample preparation, have been described extensively elsewhere $[1,2,4]$. This work was performed in accordance with an animal care protocol approved by the University of Guelph Animal Care Committee (where husbandry, and organ harvesting of the samples took place), on behalf of the Canadian Council on Animal Care.

In humans, a diet rich in sulfur has been associated with homocystinuria, a condition which impairs the conversion of methionine to cysteine. Observable clinical conditions include marfanoid habitus, lens dislocation, arterial or venous thromboses and thromboembolism, mental retardation, and the early onset of generalized osteoporosis. Vertebral compression fractures may occur by the teenage years. Homocystinuric patients have mild joint contractures rather than the hyperextensibility seen in Marfan's syndrome. While these clinical findings are striking in young individuals, when less prominent, they may be overlooked in adults. An approximate $40 \%$ increase in the relative probability of femoral fracture has been reported in homocystinuric patients [5]. Studies in animals and in humans indicate that elevated levels of homocysteine in bone may alter collagen cross-links, and interfere with bone remodeling [6]. Whitting et al. reported osteopenia and hypercalciuria in sulfur-fed rats, in addition to loss of calcium and increased resorption in bony structures. They suggested that the effects were caused by a reaction between homocysteine and the aldehyde groups of collagen, mimicking homocystinuric osteoporosis [7].

Energy dispersive spectroscopy (EDS) is an analytical technique associated with electron microscopy, in which advantage is taken of the microscope's electron beam. In this technique, semi elastic electronelectron interactions between the microscope's electron beam and orbital electrons from sample atoms, produce electron vacancies. These are promptly filled by higher energy electrons, resulting in the emission of characteristic X-rays [8]. These X-rays are detected and compared to a stored library, which after processing produces an elemental breakdown of the sample on a fractional mass basis.

\section{Experimental Methods}

In previous work, electron microscopy studies followed by energy dispersive spectroscopy (EDS) of one sample of the experimental (slagfed), and one sample of the control (normal diet) group, were undertaken [2]. Electron micrographs of mid-diaphysis cortical tibio-tarsi of the representative samples (one each) from the control and experimental groups respectively, were obtained (JEOL model 6400 Scanning Electron Microscope, equipped with Energy Dispersive Spectroscopy (EDS) capabilities, JEOL Corp., Tokyo, Japan). Image acquisition parameters were: accelerating voltage $30 \mathrm{kV}$, beam current $1.0 \times 10^{-11} \mathrm{~A}$. Successive trials established that no special sample preparation techniques were deemed necessary, thus no metallic coating was applied to the samples. Both samples were subjected to energy dispersive spectroscopy (EDS) during imaging. The rectangles in the micrographs (Fig. 1) are the sample areas interrogated for EDS analysis.

\section{Results and Discussion}

EDS analysis (areas inside the rectangles) revealed the uniform presence of elemental sulfur at a mean concentration of $2.61 \pm 0.02 \%$ by mass fraction in the experimental sample, with no sulfur levels detected in the control sample. The detectability limit of the apparatus is quoted by the manufacturer as $0.01 \pm 0.001 \%$ by mass fraction. This correlates well with the reported $2.50 \pm 0.50 \%$ sulfur concentration by mass fraction reported for nickel recovery slag in the Sudbury basin [3]. 

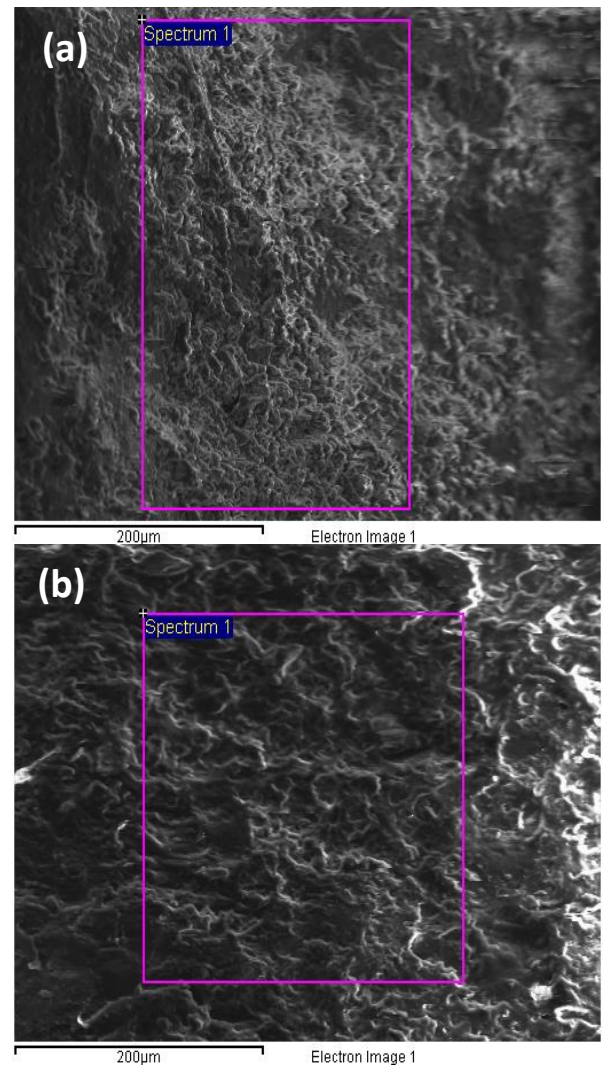

Fig. 1 a) Electron micrographs of cortical tibio-tarsi of a representative sample from the control group (normal diet) and b) experimental group (slag-fed) (Areas in rectangles were interrogated for EDS)

Table 1 EDS measurements of the elemental composition by mass fractions of control group sample (Fig. 1a). Note the absence of sulfur

\begin{tabular}{llllll}
\hline Spectrum & In stats. & 0 & P & Ca & Total \\
\hline Spectrum 1 & Yes & 70.44 & 13.59 & 15.98 & 100.00 \\
Mean & & 70.44 & 13.59 & 15.98 & 100.00 \\
Std. deviation & & 0.00 & 0.00 & 0.00 & \\
Max. & & 70.44 & 13.59 & 15.98 & \\
Min. & & 70.44 & 13.59 & 15.98 & \\
\hline
\end{tabular}

Table 2 EDS measurements of the elemental composition by mass fractions of experimental group sample (Fig. 2a). Note the presence of sulfur $(2.61 \%$ by mass fraction)

\begin{tabular}{llllll}
\hline Spectrum & In stats. & 0 & P & S & Ca \\
\hline Spectrum 1 & Yes & 80.45 & 8.33 & 2.61 & 9.65 \\
Mean & & 80.45 & 8.33 & 2.61 & 9.65 \\
Std. deviation & & 0.00 & 0.00 & 0.00 & 0.00 \\
Max. & & 80.45 & 8.33 & 2.61 & 9.65 \\
Min. & & 80.45 & 8.33 & 2.61 & 9.65 \\
\hline
\end{tabular}

Osteopenia - a homocystinuric symptom - as measured by a reduced bone mineral density (BMD), has been reported in avian species fed a sulfur-rich diet, by Ward et al. and Galiano et al. [9,2]. Another hallmark of homocystinuria is the reduction of calcium levels in bones. Utilizing mass spectrometric methods, De Vernejoul et al. reported reduced bone calcium levels in porcines subjected to a sulfur rich diet, and Galiano et al. reported similar results in pigeons $[10,2]$.

Another clinical condition associated with elevated levels of sulfur dietary intake is the loss of the mechanical integrity of long bones such as the femur. This integrity can be quantified in two ways: the breaking strength, and Young's modulus. Breaking strength is the minimum axially applied force necessary to produce failure, and Young's modulus is the ratio of stress to strain under (compressive) axial loading. Young's modulus can be thought of as the physical equivalent for a solid, of Hooke's constant for a spring - and is thus a measure of the elasticity of the solid. Reports exist of reduced breaking strength in bones of animals fed a sulfur-rich diet, mimicking homocystinuric symptoms.

For example, Huyghebaert et al. reported a reduction in breaking strength of the femora in broilers fed a fluoride and sulfur rich diet [11].
In previous work, we reported a $27 \%$ reduction in the breaking strength of tibiotarsal bones in pigeons fed a sulfur rich diet [2]. Another generally equivalent hallmark of homocystinuria proposed by Zhang and Coon, is a loss of bone elasticity as measured by a reduction in Young's modulus [12] In our own previous work, we reported a 19\% reduction in Young's modulus in bones of pigeons fed a sulfur rich diet [2].

Whitting et al. reported increased bone resorption in sulfur-fed rats, and suggested that the effects were caused by a reaction between homocysteine and the aldehyde groups of collagen, mimicking homocystinuric osteoporosis [7]. Brissot et al. described generally similar changes in bony structures in humans subjected to dietary iron and sulfur overload, conditions similar to those our experimental sample was subjected to [13]. Based on electron microscopy analysis, we reported results consistent with those of Whitting and Brissot [2]. Electron micrographs of cortical tibio-tarsi of a representative sample from the control group (normal diet) on left, and experimental group (slag diet) on right are presented in Fig. 1. Loss of granular architecture in the experimental sample is consistent with bone resorption. In particular, the typically larger cavities observed are consistent with resorptive cavities lined with osteoclasts.

\section{Conclusion}

The capability of electron microscopy-linked energy dispersive spectroscopy (EDS) to detect and quantify sulfur uptake in the tibio-tarsal bones of pigeons subjected to a nickel recovery slag-based diet, rich in elemental sulfur- was demonstrated. Specifically, EDS revealed the uniform presence of elemental sulfur in the experimental group sample, at levels closely matching the known concentration in slag; no sulphur was detected in the control sample examined. A sulfur-rich diet is associated with homocystinuria, a condition which impairs the conversion of methionine to cysteine. Among the bony changes associated with the condition are osteopenia measured by bone densitometry, loss of calcium as determined by mass spectrometry, compromised mechanical integrity as measured by reduced breaking strength and Young's modulus, and the appearance of foci of resorption as detected by conventional or electron microscopy. In previous work, we have detected and quantified all of these changes, and thus propose that our previous work along with the results of this present work, support the conclusion that a sulfur-rich, slag-based diet, can induce homocystinuria in avian species.

\section{References}

11 G.H. Parker, G. Van Der Kraak, Use of metal-rich slag as a source of grit and its effects on pigeon health and fitness, J. Env. Sci. Pollut. Res. 4(2) (2018) 256267.

[2] E. Galiano, M. Lapointe, Mechanical, chemical, structural, and radiological changes in pigeon bone, associated with the dietary intake of nickel recovery slag, J. Env. Sci. Pollut. Res. 5(2) (2019) 348-351.

[3] NSL, The leaching of slag from blast furnace, National Slag Limited, USA, 1985, pp.7-8.

[4] M. Lapointe, E. Galiano, The use of different statistical metrics to detect physiological changes in metal-overloaded avian bone, J. Env. Sci. Pollut. Res. 6(1) (2020) 412-416.

[5] L.A. Frassetto, K.M. Todd, R.C. Morris, A. Sebastian, Estimation of net endogenous noncarbonic acid production in humans from diet potassium and protein contents, Am. J. Clin. Nutr. 68 (1998) 576-583.

[6] S.R. Zwart, J.E. Davis-Street, D. Paddon-Jones, A.A. Ferrandoet, Amino acid supplementation alters bone metabolism during simulated weightlessness, Appl. Physiol. 99 (2005) 134-140.

[7] S.J. Whiting, H.H. Draper, Effect of a chronic acid load as sulfate or sulfur amino acids on bone metabolism in adult rats, Jour. Nutr. 111 (1981) 1721-1726.

[8] J. Goldstein, D.E. Newbury, D.C. Joy, C.E. Lyman, P. Echlin, et al., Scanning electron microscopy and X-ray microanalysis, Springer, USA, 2003, pp.292294.

[9] R.J. Ward, T.C. Iancu, G.M. Henderson, K.R. Kirkwood, T.J. Peters, Hepatic iron overload in birds: analytical and morphological studies, Avian Pathol. 17 (1988) 451-464.

[10] M.C. De Vernejoul, A. Pointillart, C.C. Golenzer, Effects of iron overload on bone remodeling in pigs. Am. J. Pathol. 116 (1984) 377-383.

[11] G. Huyghebaert, G. De Groote, R. Froyman, J. Derijcke, Effect of dietary fluoride on performance and bone characteristics of broilers and the influence of drying and defatting on bone-breaking strength, Poul. Sci. 67(6) (1988) 950-955.

[12] B. Zhang, C.N. Coon, The relationship of various tibia bone measurements in hens, Poul. Sci. 76(12) (1997) 1698-1701.

[13] P. Brissot, M. Ropert, C. Le Lan, O. Loréal, Non-transferrin bound iron: a key role in iron overload and iron toxicity, Biochim. Biophys. Acta 1820(3) (2012) $403-$ 410 . 